\title{
UNIQUE CONTINUATION FOR FRACTIONAL SCHRÖDINGER OPERATORS IN THREE AND HIGHER DIMENSIONS
}

\author{
IHYEOK SEO \\ (Communicated by Joachim Krieger)
}

\begin{abstract}
We prove the unique continuation property for the differential inequality $\left|(-\Delta)^{\alpha / 2} u\right| \leq|V(x) u|$, where $0<\alpha<n$ and $V \in L_{\text {loc }}^{n / \alpha, \infty}\left(\mathbb{R}^{n}\right)$, $n \geq 3$.
\end{abstract}

\section{INTRODUCTION}

In this note we are concerned with the unique continuation property for solutions of the differential inequality

$$
\left|(-\Delta)^{\alpha / 2} u\right| \leq|V(x) u|, \quad x \in \mathbb{R}^{n}, \quad n \geq 2,
$$

where $(-\Delta)^{\alpha / 2}, 0<\alpha<n$, is defined by means of the Fourier transform $\mathcal{F} f(=\widehat{f})$ :

$$
\mathcal{F}\left[(-\Delta)^{\alpha / 2} f\right](\xi)=|\xi|^{\alpha} \widehat{f}(\xi) .
$$

In particular, the equation $\left((-\Delta)^{\alpha / 2}+V(x)\right) u=0$ has attracted interest from quantum mechanics in the case $1<\alpha<2$ as well as the case $\alpha=2$. Recently, by generalizing the Feynman path integral to the Lévy one, Laskin [5] introduced the fractional quantum mechanics in which it is conjectured that physical realizations may be limited to $1<\alpha<2$, where averaged quantities are finite, and the fractional Schrödinger operator $(-\Delta)^{\alpha / 2}+V(x)$ plays a central role. Of course, the case $\alpha=2$ becomes equivalent to an ordinary quantum mechanics.

The unique continuation property means that a solution of (1.1) which vanishes in an open subset of $\mathbb{R}^{n}$ must vanish identically. In the case of $\alpha=2$, Jerison and Kenig [1] proved the property for $V \in L_{\text {loc }}^{n / 2}, n \geq 3$. An extension to $L_{\text {loc }}^{n / 2, \infty}$ was obtained by Stein [9] with small norm in the sense that

$$
\sup _{a \in \mathbb{R}^{n}} \lim _{r \rightarrow 0}\|V\|_{L^{n / 2, \infty}(B(a, r))}
$$

is sufficiently small. Here, $B(a, r)$ denotes the ball of radius $r>0$ centered at $a \in \mathbb{R}^{n}$. These results later turn out to be optimal in the context of $L^{p}$ spaces $(2,3])$.

On the other hand, the results when $\alpha \neq 2$ are rather scarce. Laba [4] considered the higher orders where $\alpha / 2$ are integers, and obtained the property for $V \in L_{\text {loc }}^{n / \alpha}$. Recently, there was an attempt [7] to handle the non-integer orders when $n-1 \leq$ $\alpha<n, n \geq 2$, from which it turns out that the condition $V \in L^{p}, p>n / \alpha$, is sufficient to have the property. Hence this particularly gives a unique continuation

Received by the editors September 5, 2013.

2010 Mathematics Subject Classification. Primary 35B60; Secondary 35J10.

Key words and phrases. Unique continuation, Schrödinger operators. 
result for the fractional Schrödinger operator in the full range $1<\alpha<2$ when $n=2$. Our aim here is to fill the gap, $0<\alpha<n-1$, for $n \geq 3$, which allows us to have the unique continuation for the fractional Schrödinger operator when $n \geq 3$ with the full range of $\alpha$.

Theorem 1.1. Let $n \geq 3$ and $0<\alpha<n$. Assume that $V \in L_{\mathrm{loc}}^{n / \alpha, \infty}$ and $u$ is a non-zero solution of (1.1) such that

$$
u \in L^{1} \cap L^{p, q} \quad \text { and } \quad(-\Delta)^{\alpha / 2} u \in L^{q},
$$

where $p=2 n /(n-\alpha)$ and $q=2 n /(n+\alpha)$. Then it cannot vanish in any non-empty open subset of $\mathbb{R}^{n}$ if

$$
\sup _{a \in \mathbb{R}^{n}} \lim _{r \rightarrow 0}\|V\|_{L^{n / \alpha, \infty}(B(a, r))}
$$

is sufficiently small. Here, $L^{p, q}$ denotes the usual Lorentz space.

Remarks. (a) The smallness condition (1.3) is trivially satisfied for $V \in L_{\text {loc }}^{n / \alpha}$ because $L_{\text {loc }}^{n / \alpha} \subset L_{\text {loc }}^{n / \alpha, \infty}$. Hence the above theorem can be seen as natural extensions to (1.1) of the results obtained in [1,9] for the Schrödinger operator $(\alpha=2)$. As an immediate consequence of the theorem, the same result also holds for the stationary equation

$$
\left((-\Delta)^{\alpha / 2}+V(x)\right) u=E u, \quad E \in \mathbb{C},
$$

because $(-\Delta)^{\alpha / 2} u=(E-V(x)) u$ and the condition (1.3) is trivially satisfied for the constant $E$.

(b) The index $n / \alpha$ is quite natural, in view of the standard rescaling: $u_{\varepsilon}(x)=u(\varepsilon x)$ takes the equation $(-\Delta)^{\alpha / 2} u=V u$ into $(-\Delta)^{\alpha / 2} u_{\varepsilon}=V_{\varepsilon} u_{\varepsilon}$, where $V_{\varepsilon}(x)=$ $\varepsilon^{\alpha} V(\varepsilon x)$. So, $\left\|V_{\varepsilon}\right\|_{L^{p, \infty}}=\varepsilon^{\alpha-n / p}\|V\|_{L^{p, \infty}}$. Hence the $L^{p, \infty}$ norm of $V_{\varepsilon}$ is independent of $\varepsilon$ precisely when $p=n / \alpha$.

(c) When $\alpha=n$ in (1.1), there are some unique continuation results with $V \in L_{\text {loc }}^{p}$, $p>1$. (See [1] and [6] for $\alpha=2$ and $\alpha=2 m(m \in \mathbb{N})$, respectively.)

\section{Proof OF THE THEOREM}

From now on, we will use the letter $C$ to denote a constant that may be different at each occurrence.

Without loss of generality, we need to prove that $u$ must vanish identically if it vanishes in a sufficiently small neighborhood of zero.

Our proof is based on the following Carleman estimate which will be shown below: If $f \in C_{0}^{\infty}\left(\mathbb{R}^{n} \backslash\{0\}\right)$ and $(-\Delta)^{\alpha / 2} f \in C_{0}^{\infty}\left(\mathbb{R}^{n} \backslash\{0\}\right)$, then there is a constant $C$ depending only on $\delta_{t}:=\min _{k \in \mathbb{Z}}|t-k|$ and $n$ such that for $t \notin \mathbb{Z}$ with $\delta_{t}<n-\alpha$

$$
\left\||x|^{-t-n / p} f\right\|_{L^{p, q}} \leq C\left\|\left.|| x\right|^{-t+\alpha-n / q}(-\Delta)^{\alpha / 2} f\right\|_{L^{q}},
$$

where $p, q$ are given as in the theorem (i.e., $1 / p+1 / q=1$ and $1 / q-1 / p=\alpha / n$ ).

Indeed, since we are assuming that $u \in L^{1} \cap L^{p, q}$ and $(-\Delta)^{\alpha / 2} u \in L^{q}$ vanish near zero (see (1.2), (1.1)), from (2.1) (with a standard limiting argument involving a $C_{0}^{\infty}$ approximate identity), we see that

$$
\left\||x|^{-t-n / p} u\right\|_{L^{p, q}} \leq C\left\||x|^{-t+\alpha-n / q}(-\Delta)^{\alpha / 2} u\right\|_{L^{q}} .
$$


Hence,

$$
\begin{aligned}
\left\||x|^{-t-n / p} u\right\|_{L^{p, q}(B(0, r))} & \leq C\left\||x|^{-t+\alpha-n / q} V u\right\|_{L^{q}(B(0, r))} \\
& +C\left\||x|^{-t+\alpha-n / q}(-\Delta)^{\alpha / 2} u\right\|_{L^{q}\left(\mathbb{R}^{n} \backslash B(0, r)\right)}
\end{aligned}
$$

The first term on the right-hand side can be absorbed into the left-hand side as follows:

$$
\begin{aligned}
C\left\||x|^{-t+\alpha-n / q} V u\right\|_{L^{q}(B(0, r))} & \leq C\|V\|_{L^{n / \alpha, \infty}(B(0, r))}\left\||x|^{-t+\alpha-n / q} u\right\|_{L^{p, q}(B(0, r))} \\
& \leq \frac{1}{2}\left\||x|^{-t-n / p} u\right\|_{L^{p, q}(B(0, r))}
\end{aligned}
$$

if we choose $r$ small enough (see (1.3) ). Here, recall that $\alpha-n / q=-n / p$, and $\left\||x|^{-t-n / p} u\right\|_{L^{p, q}(B(0, r))}$ is finite since $u \in L^{p, q}$ vanishes near zero. So, we get

$$
\left\|(r /|x|)^{t+n / p} u\right\|_{L^{p, q}(B(0, r))} \leq 2 C\left\|(-\Delta)^{\alpha / 2} u\right\|_{L^{q}\left(\mathbb{R}^{n} \backslash B(0, r)\right)}<\infty .
$$

Now, we choose a sequence $\left\{t_{i}\right\}$ of values of $t$ tending to infinity such that $\delta_{t_{i}}$ is independent of $i \in \mathbb{N}$. Then, by letting $i \rightarrow \infty$, we see that $u=0$ on $B(0, r)$, which implies $u \equiv 0$ by a standard connectedness argument.

Proof of (2.1). We will show (2.1) using Stein's complex interpolation, as in 9], on an analytic family of operators $T_{z}$ defined by

$$
T_{z} g(x)=\int_{\mathbb{R}^{n}} K_{z}(x, y) g(y)|y|^{-n} d y,
$$

where $K_{z}(x, y)=H_{z}(x, y) / \Gamma(n / 2-z / 2)$ with

$$
H_{z}(x, y)=|x|^{-t}|y|^{n+t-z} c_{z}\left(|x-y|^{-n+z}-\left.\sum_{j=0}^{m-1} \frac{1}{j !}\left(\frac{\partial}{\partial s}\right)^{j}|s x-y|^{-n+z}\right|_{s=0}\right) .
$$

Note that for $f \in C_{0}^{\infty}\left(\mathbb{R}^{n} \backslash\{0\}\right)$

$$
T_{\alpha}\left(|y|^{-t+\alpha}(-\Delta)^{\alpha / 2} f(y)\right)(x)=|x|^{-t} f(x) / \Gamma(n / 2-\alpha / 2)
$$

(see Lemma 2.1 in []).

Let $m$ be a fixed positive integer such that $m-1<t<m$, and recall the following two estimates for the cases of $\operatorname{Re} z=0$ (Lemma 2.3 in $\left[1\right.$ ) and $n-1<\operatorname{Re} z<n-\delta_{t}$ (Lemma 4 in [9]): There is a constant $C$ depending only on $\delta_{t}$ and $n$ such that

$$
\left\|T_{i \gamma} g\right\|_{L^{2}\left(d x /|x|^{n}\right)} \leq C e^{c|\gamma|}\|g\|_{L^{2}\left(d x /|x|^{n}\right)}, \quad \gamma \in \mathbb{R},
$$

and

$$
\left\|T_{z} g\right\|_{L^{r}\left(d x /|x|^{n}\right)} \leq C e^{c|\gamma|}\|g\|_{L^{s}\left(d x /|x|^{n}\right)}, \quad \gamma=\operatorname{Im} z \in \mathbb{R},
$$

where $n-1<\beta=\operatorname{Re} z<n-\delta_{t}, 1 / s-1 / r=\beta / n$ and $1<s<n / \beta$.

We first consider the case where $n-1<\alpha<n$. Note that we can choose $\beta$ so that $\alpha<\beta<n-\delta_{t}$, since we are assuming $\delta_{t}<n-\alpha$. Hence, by Stein's complex interpolation ([8]) between (2.3) and (2.4), we see that

$$
\left\|T_{\alpha} g\right\|_{L^{r}\left(d x /|x|^{n}\right)} \leq C\|g\|_{L^{s}\left(d x /|x|^{n}\right)},
$$

where $1 / s-1 / r=\alpha / n$ and $1<s<n / \alpha$. From this and (2.2), we get

$$
\left\||x|^{-t-n / r} f\right\|_{L^{r}} \leq C\left\||x|^{-t+\alpha-n / s}(-\Delta)^{\alpha / 2} f\right\|_{L^{s}}
$$


with the same $r, s$ in (2.5), since we are assuming $(-\Delta)^{\alpha / 2} f \in C_{0}^{\infty}\left(\mathbb{R}^{n} \backslash\{0\}\right)$. Note that $1<q<n / \alpha$. So, we can choose $r_{j}, s_{j}, j=1,2$, such that

$$
1<s_{1}<q<s_{2}<n / \alpha, \quad 1 / s_{j}-1 / r_{j}=\alpha / n,
$$

and for $t_{j}=t+n\left(1 / p-1 / r_{j}\right)$

$$
m-1<t_{j}<m, \quad \delta_{t} / 2 \leq \delta_{t_{j}} \leq 3 \delta_{t} / 2 .
$$

Hence we can apply (2.6) with $t=t_{j}$ to obtain

$$
\left\||x|^{-t-n / p} f\right\|_{L^{r_{j}}} \leq C\left\||x|^{-t+\alpha-n / q}(-\Delta)^{\alpha / 2} f\right\|_{L^{s_{j}}}
$$

for $j=1,2$. Since $r_{1}<p<r_{2}$ and $s_{1}<q<s_{2}$, by real interpolation ([8]) between the estimates in (2.7), we see that for $1 \leq w \leq \infty$

$$
\left\||x|^{-t-n / p} f\right\|_{L^{p, w}} \leq C\left\||x|^{-t+\alpha-n / q}(-\Delta)^{\alpha / 2} f\right\|_{L^{q, w}} .
$$

By choosing $w=q$, we get (2.1).

Now we turn to the remaining case where $0<\alpha \leq n-1$. In this case, (2.5) is valid for $1 / s-1 / r=\alpha / n$ and

$$
\frac{1}{2}\left(1-\frac{\alpha}{n-1}\right)+\frac{\alpha}{n}<\frac{1}{s}<\frac{1}{2}+\frac{\alpha}{2(n-1)},
$$

because we can choose $\beta$ so that $n-1<\beta<n-\delta_{t}$. Since (2.8) holds for $s$ replaced by $q$, repeating the previous argument, one can show (2.1). We omit the details.

\section{ACKNOWLEDGMENT}

The author is very grateful to Luis Escauriaza for bringing the author's attention to the papers [1,9] and for helpful suggestions and discussions.

\section{REFERENCES}

[1] David Jerison and Carlos E. Kenig, Unique continuation and absence of positive eigenvalues for Schrödinger operators, Ann. of Math. (2) 121 (1985), no. 3, 463-494, DOI 10.2307/1971205. With an appendix by E. M. Stein. MR794370 (87a:35058)

[2] Carlos E. Kenig and Nikolai Nadirashvili, A counterexample in unique continuation, Math. Res. Lett. 7 (2000), no. 5-6, 625-630, DOI 10.4310/MRL.2000.v7.n5.a8. MR1809288 (2001m:35044)

[3] Herbert Koch and Daniel Tataru, Sharp counterexamples in unique continuation for second order elliptic equations, J. Reine Angew. Math. 542 (2002), 133-146, DOI 10.1515/crll.2002.003. MR:1880829(2002m:35020)

[4] Izabella Łaba, Unique continuation for Schrödinger operators and for higher powers of the Laplacian, Math. Methods Appl. Sci. 10 (1988), no. 5, 531-542, DOI 10.1002/mma.1670100504. MR965420 (89k:35063)

[5] Nikolai Laskin, Fractional quantum mechanics and Lévy path integrals, Phys. Lett. A 268 (2000), no. 4-6, 298-305, DOI 10.1016/S0375-9601(00)00201-2. MR1755089 (2000m:81097)

[6] Ihyeok Seo, Remark on unique continuation for higher powers of the Laplace operator, J. Math. Anal. Appl. 397 (2013), no. 2, 766-771, DOI 10.1016/j.jmaa.2012.08.035. MR2979611

[7] Ihyeok Seo, On unique continuation for Schrödinger operators of fractional and higher orders, Math. Nachr. 287 (2014), no. 5-6, 699-703, DOI 10.1002/mana.201300008. MR3193945

[8] Elias M. Stein, Interpolation of linear operators, Trans. Amer. Math. Soc. 83 (1956), 482-492. MR.0082586 (18,575d)

[9] E. M. Stein, Appendix to “unique continuation”, Ann. of Math. 121 (1985), 489-494.

Department of Mathematics, Sungkyunkwan University, Suwon 440-746, Republic of KOREA

E-mail address: ihseo@skku.edu 